\title{
Evaluación del programa piloto de reducción de sal/sodio en el pan en Santiago de Chile
}

\author{
Evaluation of the pilot program to reduce salt/sodium \\ in bread in Santiago of Chile
}

\begin{abstract}
Introduction: Considering the high burden of disease associated with excessive salt intake of the population, Chile initiated a pilot program between the Ministry of Health (MINSAL), the Industrial Bakers Federation (FECHIPAN) and the Chilean Association of Supermarkets $(A S A C H)$ in order to achieve a gradual reduction of salt in bread. Objective: To analyze the amount of sodium in bread samples from bakeries belonging to the program and those not participating in Santiago. Materials and methods: Cross-sectional study with random sampling of two products in five pilot and five control bakeries. Sodium was analysed by atomic absorption spectrophotometry and the mean was used for analysis (mg/100 g of bread). For comparison the Student's t test was utilized and significance was established at $p<0,05$. Results: The average sodium concentration in the control group was 597,2 $\pm 106,4 \mathrm{mg} / 100 \mathrm{~g}$ bread while in the experimental group was $600,9 \pm 106,2 \mathrm{mg} / 100 \mathrm{~g}$ bread showing no significant differences between them. There was considerable variability in the levels of sodium in both groups, with values ranging from 403 to $824 \mathrm{mg} / 100 \mathrm{~g}$. Discussion: The concentration of sodium in the bread was similar in both groups, suggesting a reduction in salt content in the control bakeries. More studies are needed to better understand the national reality in this matter.
\end{abstract}

Key words: Bread, sodium, salt, pilot program, Ministry of Health, high blood pressure.

\section{INTRODUCCIÓN}

En la actualidad las enfermedades cardiovasculares (ECV) son la primera causa de muerte tanto a nivel mundial (1) como nacional (2). La hipertensión arterial (HTA) es uno de los principales factores de riesgo para el desarrollo de las ECV (3), y a su vez, está demostrado que el consumo de sal está fuertemente asociado a los valores de presión arterial (PA). Por este hecho, distintos países del mundo, entre ellos Chile, están implementando programas para disminuir el consumo de sal/sodio en la población $(4,5)$.

Según la última estimación de ingesta de pan realizada por la Oficina de Estudios y Políticas Agrarias (ODEPA), el consumo promedio en Chile en el año 2010 fue de 86 kg/habitante, lo que representa una ingesta diaria de $253 \mathrm{~g} /$ habitante al día (6).

Un estudio realizado el 2010 por el Ministerio de Salud en relación al contenido de sodio en 52 muestras de 4 variedades de pan (marraqueta, hallullas, frica y baguette) de la Región Metropolitana, mostró una concentración promedio
Karen Valenzuela L. $(1,2)$ Vilma Quitral R. (2) Beatriz Villanueva A. (3)

Fernando Zavala M. (4) Eduardo Atalah S. (2)

(1) Servicio Medicina, Hospital de Carabineros. Santiago, Chile. (2) Departamento de Nutrición, FacultaddeMedicina, Universidadde Chile. Santiago, Chile. (3) Consulta privada, Santiago, Chile. (4) Carrera de pregrado, Facultad de Medicina, Universidad Andrés Bello. Santiago, Chile.

Dirigir la correspondencia a: Karen Valenzuela 1 . Flor de Azucenas 65 dpto. 501 Las Condes Santiago. Chile

Fono: 9-75174075

E-mail: drakarenvalenzuela@gmail.com

Este trabajo fue recibido el 5 de Marzo de 2013 y aceptado para ser publicado el 5 de Mayo de 2013.

de $774 \pm 105 \mathrm{mg} / 100$, con un nivel mayor en las marraquetas (831 $\pm 105 \mathrm{mg} / 100 \mathrm{~g})$ en relación a las hallullas (735 \pm 92 $\mathrm{mg} / 100 \mathrm{~g})$ (datos no publicados).

En función de estos antecedentes en septiembre del año 2010 el MINSAL junto con la FECHIPAN y la ASACH acordaron disminuir en forma paulatina la concentración de sal agregada en la fabricación de los tipos de pan hallulla y marraqueta (7). La iniciativa comenzó con un programa piloto que incluyó 100 panaderías a lo largo del país, pero con el compromiso por parte de FECHIPAN y de ASACH de incorporar al menos 500 panaderías para fines del 2011 y el 100\% de sus asociados para el 2014 (8). Se fijó una meta de reducción de la concentración de sodio con la que se fabrica el pan desde $800 \mathrm{mg} / 100 \mathrm{~g}$ pan $\mathrm{a} \leq 500 \mathrm{mg} / \mathrm{pan}$ al año 2011 y a $\leq 400$ $\mathrm{mg} / 100 \mathrm{~g}$ al año 2014 (9).

El objetivo de este estudio es analizar la concentración de sodio presente en muestras de pan, tanto de panaderías adheridas al programa como panaderías no participantes de éste. 


\section{MATERIALES Y MÉTODOS}

Diseño: Estudio transversal analítico.

Universo: 95 panaderías participantes del programa MINSAL-FECHIPAN-ASACH y las panaderías no adheridas, en el Gran Santiago.

Muestra: Selección aleatoria de 5 panaderías pertenecientes al programa (grupo experimental), y 5 panaderías no adheridas (grupo control) ubicadas en las mismas comunas que las panaderías del grupo experimental. El tamaño de la muestra se calculó a partir de la información generada por el Ministerio de Salud el 2010 en relación al contenido de sodio en el pan de panaderías de la Región Metropolitana; estimando una concentración promedio de $774 \pm 105$ mg/100 g en el grupo control, una disminución del $20 \%$ en el grupo experimental, una desviación estándar de 15\%, nivel de confianza $95 \%$, poder $90 \%$ se estimó un tamaño mínimo de 9 muestras, que se aumentó a 10 para asegurar una mayor confiabilidad.

En cada una de las panaderías se adquirió una muestra de marraqueta y una de hallulla, de modo que se obtuvieron 10 muestras para el grupo control y 10 para el grupo experimental.

Las muestras se congelaron y almacenaron a $-20^{\circ} \mathrm{C}$ y se transportaron hacia al laboratorio externo manteniendo la cadena de frío.

Análisis del sodio: Se determinó la concentración de sodio del pan en un laboratorio externo acreditado, por medio de la técnica de espectrofotometría de absorción atómica 985.35 (AOAC, 1995), la cual consiste el secar el producto hasta obtener sus cenizas, diluirlas en ácido nítrico y luego realizar una curva de calibración/concentración/absorbancia con un estándar. La curva obtenida para el sodio debe ser leída a una longitud de onda de $589 \mathrm{~nm}$ (10). Los resultados se expresaron en $\mathrm{mg}$ de sodio/100 $\mathrm{g}$ pan.

Análisis de datos: Los datos fueron analizados con el programa STATA 11.1. Se analizó la normalidad de la concentración de sodio en el pan y como tuvo una distribución normal se utilizó el promedio y desviación estándar como medidas de tendencia central. Para comparación de ambos grupos se utilizó la prueba de T de Student, considerando como significativo un valor $\mathrm{p}<0,05$.

\section{RESULTADOS}

Tanto para el grupo experimental como para el grupo control el promedio de la concentración total de sodio fue cercano a los $600 \mathrm{mg} / 100 \mathrm{~g}$ de pan, no observándose diferencias significativas entre ellos $(p=0,9)$ (tabla 1$)$.

$\mathrm{Al}$ analizar por tipo de pan, se observó una mayor concentración de sodio en la hallulla del grupo experimental, aunque sin diferencias significativas entre ambos grupos. En el pan marraqueta, se obtuvo una mayor concentración de sodio en el grupo control, aunque las diferencias tampoco fueron estadísticamente significativas (tabla 1).

Aún cuando el coeficiente de variación fue relativamente bajo, destaca una importante variabilidad en ambos grupos, con valores máximos y mínimos de concentración de sodio en el grupo intervenido de 497 y 824 mg/100 g y algo menores en el grupo control (403 y $768 \mathrm{mg} / 100 \mathrm{~g}$ ).

\section{DISCUSIÓN}

El pan evaluado presenta en promedio alrededor de 600 mg sodio/ 100 g de pan, cantidad cercana a la reducción del sodio en el pan propuesta por el MINSAL para las panaderías intervenidas durante el desarrollo del programa (9). Sin embargo, sorprendentemente el promedio de concentración de sodio, en ambos grupos fue prácticamente el mismo, presentándose la paradójica situación en que el grupo control no se comportó como tal.

Para interpretar los resultados debe considerarse el reducido número de panaderías y de muestras de pan en cada panadería analizada, lo que podría entregar información no necesariamente extrapolable al universo en estudio. A pesar de esta limitación, el análisis demostró que se está cumpliendo el acuerdo voluntario con el Ministerio de Salud, aunque con un comportamiento heterogéneo entre las panaderías. Ello concuerda con lo observado en la fiscalización de locales adheridos al programa, realizada en el primer semestre del año 2012 por la Seremi de Salud en la cual se constató una disminución aproximada de 200 mg de sodio en $100 \mathrm{~g}$ de pan (11). Ambos resultados demuestran una tendencia hacia el cumplimiento de los acuerdos fijados por parte de las panaderías del grupo experimental, pero lo interesante y sorprendente es la tendencia hacia la fabricación de un pan más saludable en aquellos locales no adheridos al acuerdo.

Lo anteriormente señalado podría deberse a un firme compromiso de las panaderías participantes del programa, así como también el deseo por parte de FECHIPAN y todas sus panaderías pertenecientes de contribuir, por medio de sus productos, a disminuir el consumo de sal de todos los chilenos independientemente del acuerdo realizado con el MINSAL (12).

En la fabricación del pan, la sal no sólo le otorga el sabor salado, sino que también optimiza el sabor de la masa, mejora sus características organolépticas y reológicas, disminuye la actividad de la levadura reduciendo la producción de gas, controla la microflora de la masa limitando la actividad de

\section{TABLA 1}

Promedio de la concentración de sodio (mg/100 g pan) según tipo de pan y grupo estudiado.

\begin{tabular}{|c|c|c|c|c|}
\hline & $\begin{array}{c}\text { Grupo intervenido } \\
\text { XDE }\end{array}$ & $\begin{array}{c}\text { Grupo control } \\
\text { X DE }\end{array}$ & $\begin{array}{l}\text { Total } \\
X \mathrm{DE}\end{array}$ & $p^{*}$ \\
\hline Hallulla & $636,4 \quad 124,1$ & $580,8 \quad 107,7$ & $608,6 \quad 113,0$ & 0,47 \\
\hline Marraqueta & $558,0 \quad 79,1$ & $621,0 \quad 113,0$ & $589,5 \quad 97,8$ & 0,33 \\
\hline Total & $600,9 \quad 106,2$ & $597,2 \quad 106,4$ & $599,0 \quad 103,5$ & 0,94 \\
\hline $\mathrm{p}$ & 0,26 & 0,69 & 0,93 & \\
\hline
\end{tabular}


microorganismos, e inhibe la acción de proteínas proteolíticas por lo que se refuerza el gluten (13).

Si bien una menor utilización de la sal en fabricación del pan resulta beneficiosa para la población, para la industria representa un gran desafío pues este mineral es importante para obtener un producto de buena calidad y aceptabilidad en los consumidores.

Para lograr fabricar un pan más saludable se ha propuesto que la disminución de la sal agregada al pan sea progresiva, de modo que los consumidores se adapten en forma gradual a este nuevo producto. Estudios internacionales han indicado buenos resultados de aceptabilidad del sabor del pan con disminuciones paulatinas de la sal en su fabricación (14, 15), aunque aún no existe un consenso sobre un "limite de seguridad" de concentración de sal en el pan en la cual no se vea afectada la aceptabilidad de éste. En nuestro país, en un artículo técnico elaborado por Granotec, se indica que $67 \%$ de los encuestados prefiere un pan con menos sal (aunque no se indica la concentración de sal) versus un pan normal, mientras que un $8 \%$ no nota la diferencia en entre ambos tipos de pan (16).

En Australia y Nueva Zelanda disminuciones aún mayores que en Chile, ya han sido publicadas. En Australia entre los años 2007-2010 la proporción de panes con un contenido de sodio menor a $400 \mathrm{mg} / 100 \mathrm{~g}$ aumentó de $25 \%$ a 50\%. En Nueva Zelanda en el mismo período el aumento fue de $0 \%$ a 17\% (17). En el Reino Unido por su parte, información al año 2010 indica que el contenido de sodio en el pan blanco es de 397 mg/100 g/pan (18). Tales cifras exitosas sirven de ejemplo para continuar en camino de la reducción de la sal en el contenido del pan.

En Argentina en el año 2010 se implementó el programa "Menos Sal, Mas Vida" el cual con más de 6000 panaderías adheridas, logró una reducción de $25 \%$ de sal en la fabricación del pan (19). En una evaluación realizada como parte de este programa, en la provincia de la Pampa, se constató una diferencia estadísticamente significativa en la sal utilizada tanto en la fabricación del pan como otros productos de pastelería entre los años 2010 y 2011 (20).

El cumplimiento del acuerdo sobre el paulatino descenso en la cantidad de sal/ sodio en la fabricación pan es un importante aporte para una reducción de la ingesta de este mineral en la población de nuestro país, puesto que el elevado consumo de pan hace de éste una las fuentes principales de aporte de sal/sodio en la dieta. Puesto que cada unidad de pan que se consume contiene aproximadamente 1,5 g de sal, la ingesta diaria de 2 unidades aporta más de la mitad de la recomendación de consumo diario por persona establecido por la OMS (5 g/día) (7)

Dado la importancia de la reducción de consumo de sal/sodio para la salud de nuestra población, se hace necesario además poner en marcha campañas de difusión masiva sobre esta iniciativa y educar sobre la nocividad de sodio, y no solamente de la sal. Un reciente estudio llevado a cabo en 3 países de Latinoamérica indicó que una fracción de los participantes desconoce qué es el sodio y sus efectos en la salud, y lo que sería más alarmante aún, se reconoce un elevado riesgo en el consumo de sal, pero no se percibe este riesgo como real (21).

Dentro de las limitaciones de este estudio se reconoce el limitado tamaño de muestra, el cual no permite extrapolar nuestros resultados al universo de las panaderías participantes de este programa. Existe por tanto la necesidad de ampliar el estudio tanto en locales participantes como no participantes, no solo en la Región Metropolitana a modo de medir los avances de este programa en el resto del país.

\section{RESUMEN}

Antecedentes: Considerando la alta carga de enfermedades asociadas a un excesivo consumo de sal, Chile inició un programa piloto entre el Ministerio de Salud (MINSAL), la Federación de Industriales Panaderos (FECHIPAN) y la Asociación Chilena de Supermercados (ASACH) con el propósito de lograr una disminución paulatina de la sal con que se fabrica el pan. Objetivo: Analizar la concentración de sodio (mg/100 g) en muestras de pan de panaderías adheridas al programa y panaderías no participantes, del Gran Santiago. Materiales y métodos: Estudio transversal analítico; muestreo aleatorio de dos muestras de pan en cinco panaderías del programa piloto y cinco panaderías control. Análisis de sodio por espectrofotometría de absorción atómica y determinación del promedio de éste en las muestras ( $\mathrm{mg} / 100 \mathrm{~g}$ de pan). Para la comparación de promedios se utilizó t de Student, considerando significativo un $p<0,05$. Resultados: La concentración promedio de sodio en el pan en el grupo control fue 597, $2 \pm 106,4 \mathrm{mg} / 100 \mathrm{~g}$ y en el grupo intervenido $600,9 \pm 106,2 \mathrm{mg} / 100 \mathrm{~g}$, sin diferencias significativas entre ellos. Existe bastante variabilidad en los niveles de sodio en ambos grupos, con valores extremos de 403 y 824 mg/100 g. Discusión: La concentración de sodio en el pan fue similar en ambos grupos. La reducción del sodio en panaderías no participantes en el programa, sugiere preocupación de la industria por fabricar un pan más saludable. Son necesarios estudios con mayor representatividad para conocer mejor la realidad nacional.

Palabras clave: Pan, sodio, sal, programa piloto, Ministerio de Salud, hipertensión arterial.

Conflictos de interés: Todos los autores declaran no tener conflictos de interés con las empresas o instituciones vinculadas con el tema estudiado.

\section{BIBLIOGRAFÍA}

1. Enfermedades cardiovasculares. Datos principales. OMS. Disponible en: http://www.who.int/mediacentre/factsheets/fs317/es/index.htm/ Fecha consulta: 03/05/13.

2. Estadisticas vitales INE 2007. Disponible en: http://www. ine.cl/canales/chile_estadistico/demografia_y_vitales/estadisticas_vitales/2010/04_01_10/vitales2007.pdf Fecha consulta: 03/05/13.

3. Lombera F, Barros V, Soria F, Placer L, et al. Guías de practica clínica de la Sociedad Española de Cardiología en Hipertensión Arterial. Rev Esp Cardiol 2000; 53: 66-90.

4. Consensus Action on Salt and Health. Disponible en: http://www.actiononsalt.org.uk/about/index.htm/ Fecha consulta: 03/05/13

5. El consumo de sal en Europa. Consejo Europeo de Información sobre la Alimentación (EUFIC). Disponible en: http://www.eufic.org/article/es/artid/El-consumo-de-salen-Europa/ Fecha consulta: 03/05/13.

6. Consumo aparente de alimentos en Chile. Disponible en : http://www.odepa.gob.cl/odepaweb/publicaciones/ doc/7004.pdf Fecha consulta: 03/05/13

7. Protección de Salud. Estrategia de Reducción Sal/Sodio en los Alimentos. Ministerio de Salud de Chile. Disponible en: http://www.redsalud.gov.cl/portal/url/page/ minsalcl/g_proteccion/g_alimentos/reduccion_sodio.html Fecha consulta: 03/05/13.

8. Carta compromiso para la reducción del sal/sodio en el pan, 
MINSAL; FECHIPAN; ASACH. Disponible en: http://wWw. asach.com/CARTA\%20COMPROMISO_sodio.pdf Fecha consulta: 03/05/13.

9. Balance de gestión integral año 2011. Ministerio de Salud de Chile. Subsecretaría de Salud Pública. Disponible en: http://www.senado.cl/site/presupuesto/ cumplimiento/Otras\%20leyes/Ley\%20N\%B0\%20 $1.263 \% 20$ Administraci\% F3n\%20Financiera $\% 20$ del\%20Estado\%202012/ORD.\%201556\%20 Salud/SUBSECRETAR\% CDA\%20DE\%20SALUD\%20 P\%DABLICA.pdf Fecha consulta: 03/05/13.

10. A.O.A.C. Official Methods of Analysis of Official Analytical Chemist. 18th Edition. Horwitz W. \& Latimer G.W. Editors. U.S.A., 2005.

11. Seremi de Salud: Sal en el pan disminuyó casi 200 miligramos en promedio. Año 2012. Disponible en: http:// www.cooperativa.cl/prontus_nots/site/artic/20120626/ pags/20120626162539.htm/ Fecha consulta: 03/05/13.

12. Disminución de la sal en el pan: aires de autorregulación. Disponible en: http://www.fechipan.cl/pdf/sal2.pdf Fecha consulta: 03/05/13.

13. Technologies of salt reduction in bread: issues, problems and solutions. Seminar: Salt in Bread Oct., 2009, Brussels. Disponible en: http://ec.europa.eu/health/nutrition_physical_activity/docs/ev20091021_salovaara_en.pdf Fecha consulta: 03/05/13.

14. Belz MC, Ryan LA, Arendt EK. The impact of salt reduction in bread: a review. Crit Rev Food Sci Nutr. 2012; 52:
514-24.

15. Girgis S, Neal B, Prescott J, Prendergast J, Dumbrell S, Turner $C$, Woodward M. A one-quarter reduction in the salt content of bread can be made without detection. Eur J Clin Nutr. 2003; 57(4):616-20.

16. Reducción de sal en el pan. Disponible en http://www. indupan.cl/sal.html. Fecha de consulta 03/05/13.

17. Dunford EK, Eyles H, Mhurchu CN, Webster JL, Neal BC. Changes in the sodium content of bread in Australia and New Zealand between 2007 and 2010: implications for policy. Med J Aust. 2011; 195(6):346-9.

18. Webster JL, Dunford EK, Neal BC. A systematic survey of the sodium contents of processed foods. Am J Clin Nutr. 2010; 91(2):413-2.

19. Campaña "Menos Sal, Mas Vida". Información. Ministerio de Salud de Argentina. Disponible en: http://www. msal.gov.ar/ent/images/stories/ciudadanos/pdf/201207_acuerdos-voluntarios-industria-reduccion-sodio.pdf Fecha consulta: 03/05/13.

20. Acuerdos voluntarios para reducción progresiva de sodio de los alimentos industrializados, año 2011. Disponible en : http://msal.gov.ar/ent/MenosSalMasVida/PDF/Presentacion.pdf Fecha de consulta: 03/05/13.

21. Sánchez G, Peña L, Varea S, Mogrovejo P, Goetschel ML, et al. Conocimientos, percepciones y comportamientos relacionados con el consumo de sal, la salud y el etiquetado nutricional en Argentina, Costa Rica y Ecuador. Rev Panam Salud Pública 2012; 32: 259-64. 